\title{
Protein labeling approaching its PRIME
}

To site-specifically label proteins in living cells, researchers mutate an enzyme to recognize a small fluorophore and catalyze its attachment to a small peptide tag.

Fluorescent protein fusions have long been the go-to tools for labeling proteins inside live cells. But a substantial drawback of this genetic fusion approach, as well as of post-translational labeling methods - the large size of the added tag- limits their utility for some studies. Live-cell labeling with the small dye FlAsH has been effective for highly expressed proteins or for oligomeric proteins but is otherwise impeded by high background signal.

Pursuing an enzyme-mediated labeling approach, Alice Ting's group at the Massachusetts Institute of Technology was trying to alter an enzyme so that it would ligate a fluorophore instead of its natural ligand. But the enzyme they were using, biotin ligase, was too specific for its smallmolecule substrate, biotin, even after activesite mutagenesis. In a literature search, they came across a structurally homologous but considerably more promiscuous enzyme, the Escherichia coli lipoic acid ligase (LplA). They had previously shown that instead of its natural substrate, LplA can ligate an alkyl azide, which can be subsequently derivatized with a fluorophore in a second step. Using this approach, they labeled cell-surface proteins (Fernández-Suárez et al., 2007).

To get this to work inside cells, Ting recalls, "we wanted to simplify everything and have a one-step protocol, and we started thinking about having a direct fluorophore ligase." Thus, they performed mutagenesis to create an enzyme that would incorporate coumarin-one of the smallest fluorophores useful for live-cell imaging. They zoomed in on tryptophan 37 in the lipoic acid binding tunnel. Two different substitutions at this position-W37V or W27I-resulted in 'fluorophore ligases' that could attach the coumarin to a 13-amino-acid LplA recognition sequence (Uttamapinant et al., 2010). In live HEK, HeLa and COS-7 cells, both of the selected mutant enzymes specifically labeled acceptor peptide-target protein fusions.
Named 'probe incorporation mediated by enzymes' (PRIME), this method is straightforward and nontoxic to cells. "It's transfect with the ligase and peptide fusion constructs, add the dye and change the medium. There is basically no optimization," says Ting. And PRIME is not plagued by the high background signal of FlAsH labeling. As a proof of principle, her group used PRIME to label nuclear actin and detected both monomeric and filamentous actin. Uniquely, the PRIME technology can be used to genetically target the fluorophore ligase to a cellular compartment by means of a localization signal sequence to selectively label the target proteins therein.

"Compared to GFP, which is what everyone is using now," Ting says, this coumarinbased PRIME "offers a size advantage, but not a photophysical benefit because most fluorescent proteins are brighter than coumarin. But the two-step version of PRIME with an alkyl azide substrate lets you label with any fluorophore structure that you want" because the fluorophore does not have to fit in the ligase active site. Ting's group is now working on a two-step labeling approach with ATTO dyes. Though these dyes are larger than coumarin, they are very bright and much better suited for single-molecule and super-resolution imaging applications.

As for labeling in other types of cells, Ting says, "we still need to test it, but it will probably work in yeast. In bacteria, there is a fundamental limitation because there are substrates of [LplA] that probably cross-react with our coumarin ligase, so we will have background." To solve that problem, she hopes to engineer a ligase that does not recognize anything resembling the natural substrate.

With these enhancements, PRIME is surely set to become an integral part of the imaging toolbox.

\section{Irene Kaganman}

\section{RESEARCH PAPERS}

Fernández-Suárez, M. et al. Redirecting lipoic acid ligase for cell surface protein labeling with smallmolecule probes. Nat. Biotechnol. 25, 1483-1487 (2007).

Uttamapinant, C. et al. A fluorophore ligase for sitespecific protein labeling inside living cells. Proc. Natl. Acad. Sci. USA 107, 10914-10919 (2010). 